\title{
AN UPDATED SIMULATION FRAMEWORK FOR THE PREDICTION OF PROCESS INDUCED SHAPE DISTORTION IN THERMOSET COMPOSITES.
}

\author{
Neoklis Traiforos ${ }^{1,2}$, Thomas Turner ${ }^{2}$, Patrik Runeberg ${ }^{3}$, Daoud Fernass ${ }^{1}$, Dimitrios
} Chronopoulos $^{2}$, Florian Glock ${ }^{1}$, Gerd Schuhmacher ${ }^{1}$

${ }^{1}$ Airbus Defence and Space GmbH, Rechliner Str., 85077, Manching, Germany

${ }^{2}$ The University of Nottingham, Faculty of Engineering, Polymer Composites Research Group, Advanced Manufacturing Building, 522 Derby Rd, Nottingham NG8 1BB, United Kingdom

${ }^{3}$ Premium AEROTEC GmbH, Haunstetter Str. 225, 86179, Augsburg, Germany

* neoklis.traiforos@airbus.com

A significant problem encountered during the manufacturing process of thermoset composite structures is the distortion of their shape from their CAD-nominal geometry. Shape distortions can be attributed to the residual stresses which are imposed within the structure during its manufacture.

The present study investigates the ability of two simulation processes to accurately predict process induced shape distortions of aerospace frame structures. The two simulation processes use the same geometry, mesh quality, material properties, micromechanics and cure kinetics model, but differ on the definition of their constitutive equation. The constitutive equation of the material models is implemented using the UMAT subroutine of the ABAQUS FEA software.

The first simulation process which is investigated employs the CHILE (Cure Hardening Instantaneous Linear Elastic) model [1] which is widely used because of its simplicity, its fast calculation times and its enhanced accuracy to simulate shape distortions relative to the pure elastic models. However, the CHILE model cannot predict any stress relaxation during curing.

The second simulation process which is investigated employs the Harrison Poon and M. Fouad Ahmad innovative constitutive update scheme for simulating anisotropic, thermo rheologically simple, viscoelastic solids [2]. The modelling of the viscoelastic behaviour of the resin during the curing process is done with the use of a generalized Maxwell model. The implementation of a viscoelastic material model allows the simulation of all the time dependent factors which affect shape distortions like the cure time, heating and cooling rates and stress relaxation. The effect of different mechanical boundary conditions on simulation results is also investigated for both material models.

The results of the two simulation processes are compared in terms of calculation times and regarding their ability to accurately predict shape distortions of a typical aerospace frame. As a reference for assessing the accuracy of the simulation processes and the boundary conditions, measurement data which come from the 3D scanning of the manufactured frame is used. Conclusions are made regarding the accuracy of the two material models and the effect of mechanical boundary conditions on simulation results. Further steps to increase the accuracy of the simulation processes or reduce their calculation times are proposed.

\section{References}

(1) Johnston AA. "An integrated model of the development of process-induced deformation in autoclave processing of composite structures" [Ph.D.]. Ann Arbor: The University of British Columbia (Canada), 1998.

(2) H. Poon and M. F. Ahmad, "A material point time integration procedure for anisotropic, thermo rheologically simple, viscoelastic solids“. Computational Mechanics, Vol. 21, No. 3, pp 236-242, 1998. 